\title{
Las diferentes formas de morir. Reflexiones éticas
}

\author{
A. AZULAY TAPIERO \\ Servicio de Medicina Interna. Hospital Dr. Moliner. Serra. Valencia
}

THE DIFFERENT WAYS OF DYING. ETHICAL REFLECTIONS

\begin{abstract}
RESUMEN
El proceso de la muerte se ve influenciado por multitud de factores y
RESUMEN
El proceso de la muerte se ve influenciado por multitud de factores y no es habitual que el enfermo decida sobre sus circunstancias.

En la situación de enfermedad terminal la Medicina Paliativa se considera la mejor opción.

En ocasiones la muerte se produce como consecuencia de una acción u omisión, lo que plantea dilemas éticos importantes difíciles de resolver. En este artículo se analizan las diferencias y las similitudes existentes entre la eutanasia por acción y por omisión.
\end{abstract}

PALABRAS CLAVE: Bioética. Eutanasia. Cuidados Paliativos.

\begin{abstract}
Death Process is seen influenced by multitude of factors. It's not habitual that the patient decides about the circumstances of his death.

In the terminally ill situation, the Palliative Medicine is considered the best option.

Sometimes the death is produced as consequence of an action or omission, which raises ethical important dilemmas difficult to resolve. The similarities and differences between active euthanasia and passive euthanasia are analysed.
\end{abstract}

KEY WORDS: Bioethics. Euthanasia. Palliative Care.

Azulay Tapiero A. Las diferentes formas de morir. Reflexiones éticas. An Med Interna (Madrid) 2004; 21: $355-358$.

Un principio fundamental arraigado en la Medicina desde los tiempos más remotos, desde su inicio, es el respeto a la vida humana, que obliga a hacer todo lo posible por protegerla y mantenerla en las mejores condiciones posibles. Este principio ético era asumido y no planteaba ningún dilema hasta la segunda mitad del siglo XX, momento en el que ha empezado a adquirir protagonismo la tecnología aplicada al soporte vital, que permite mantener a una persona con vida durante largos periodos de tiempo. Estos importantes avances tecnológicos han modificado el curso natural del proceso de la muerte, de modo que se podría afirmar que ésta, desde entonces, generalmente acontece por una decisión médica, por una acción u omisión, no por la naturaleza o el curso evolutivo de la enfermedad. Se puede posponer la muerte a voluntad gracias a la tecnología, lo que puede ser muy útil en determinadas circunstancias, como por ejemplo en caso de extracción de órganos para trasplante, pero en otras, evidentemente no. Esta preservación de la vida no debe verse como una obligación absoluta, ya que debe estar supeditada a que ésta sea digna y esté libre de sufrimiento. La vida, por lo tanto, no debe tener un valor absoluto y asumir esto es aceptar que en ocasiones es preferible la muerte a la vida o, por lo menos, que la muerte no es siempre la peor solución. Darle a la vida un valor absoluto equivaldría a considerar como una opción éticamente correcta la obstinación o el ensañamiento terapéutico o aplicar todas las medidas de soporte vital necesarias para mantenerla en cualquier circunstancia y durante el máximo tiempo posible, a pesar de un sufrimiento intenso.

Otra revolución importante en la Medicina, que se ha iniciado también en las últimas décadas del siglo pasado, ha sido el cambio en el modelo tradicional de la relación médicopaciente, que ha pasado del paternalismo más absoluto y bien intencionado al respeto a la autonomía del enfermo, al que se le reconocen unos derechos que le permiten estar informado

Trabajo aceptado: 21 de abril de 2004 
de su proceso patológico, si así lo desea, para poder participar en la toma de decisiones y, en última instancia, decidir sobre qué tratamientos, qué pruebas diagnósticas y qué medidas de soporte aplicar.

Estos dos cambios, en lo que respecta al proceso de la muerte cuando ésta es inevitable, obligan a:

- Reconocer la finitud de la vida humana, evitar su conservación de forma incondicional y aplicar todas las medidas necesarias y disponibles para mejorar su calidad y no su cantidad.

- Aplicar el principio ético de autonomía, que en esencia consiste en mantener al enfermo correctamente informado y respetar su criterio para que pueda participar en la toma de decisiones y pueda decidir, al menos dentro de la legalidad vigente, sobre las circunstancias de su muerte.

La muerte, cuando sobreviene por una enfermedad en la que la vida se extingue gradualmente, cuando no lo hace de forma súbita o por un proceso patológico potencialmente curable, puede acontecer de diversas maneras, algunas menos dolorosas que otras, algunas más dignas que otras, pero que en parte, el que suceda de una forma u otra, dependerá de factores sociales, económicos, culturales y políticos. En la actualidad, en nuestro ámbito, no es habitual que el enfermo decida sobre las circunstancias de su muerte, incluso a veces se le oculta su condición de moribundo, por lo que no es probable que la forma de morir dependa de sus criterios, valores y creencias.

En la situación de enfermedad terminal la muerte inevitable, como proceso, puede suceder de diversas maneras:

- La enfermedad sigue su curso natural y el paciente fallece como consecuencia de ella sin ninguna intervención sanitaria, en situación de abandono. Es infrecuente que esto suceda en una sociedad en donde la sanidad es universal y gratuita y en donde se dispone de todos los recursos sanitarios necesarios, que podrán ser bien o mal utilizados, como ocurre en nuestro ámbito. No obstante, esta forma de morir debe ser habitual en sociedades pobres, necesitadas de los medios básicos de subsistencia y, obviamente, sin una infraestructura sanitaria adecuada, como ocurre en el llamado Tercer Mundo. Es probable que en estas condiciones la muerte, aunque más aceptada, se asocie a un gran sufrimiento ya que no se dispone de los recursos necesarios para un buen control de síntomas.

- La muerte sobreviene tras aplicar durante todo el proceso patológico un tratamiento activo. El único objetivo que se plantea es la curación de las enfermedades o alargar la supervivencia. Es una forma de morir habitual en nuestro ámbito sanitario, en cualquier sala de hospitalización, en donde el personal sanitario, a medida que evoluciona la enfermedad, no entra a considerar la posibilidad de ir retirando determinadas medidas fútiles o desproporcionadas, probablemente por desconocimiento, miedo a una posible demanda (medicina defensiva) y/o falta de formación.

-El paciente fallece a pesar de la aplicación de todas las medidas farmacológicas y de soporte vital disponibles hasta el último momento. A medida que avanza la enfermedad, más agresivo es el tratamiento y más medios desproporcionados son utilizados. Es la llamada obstinación terapéutica, que considera a la muerte como un fracaso, por lo que se intenta posponer al máximo. Es frecuente en países desarrollados y con un sistema sanitario público y gratuito, como ocurre en nuestro medio. Origina un derroche excesivo desde el punto de vista económico, una mala distribución de los recursos y, lo que es peor, un sufrimiento innecesario para el enfermo.

-El paciente fallece, cuando existe un sufrimiento intenso refractario a las medidas paliativas disponibles, por la aplicación de la eutanasia activa directa y/o la ayuda al suicidio. Al menos en teoría, esta forma de llegar a la muerte sólo es posible en países donde cualquiera de estas dos prácticas está despenalizada. La legislación impone el cumplimiento de una serie de requisitos bien detallados con la finalidad de evitar posibles abusos. Es indispensable contar con el consentimiento del enfermo bien informado que ha elegido esta opción sin ninguna clase de coacción.

- La muerte se produce como consecuencia de una omisión o suspensión de medidas que prolongan la vida del enfermo. Se trata de la eutanasia pasiva. Suele ocurrir en pacientes que están en situación terminal avanzada o en coma irreversible, a los que se les suspende o no se les llega a aplicar una técnica de soporte, generalmente agresiva, sin la cual no podrían sobrevivir. Es una práctica habitual y poco cuestionada. El problema ético surge cuando el fallecimiento tiene lugar por la negación de unos cuidados que son necesarios y no desproporcionados.

- La muerte acontece en el marco de unos cuidados paliativos de calidad, en donde el objetivo es conseguir una buena muerte y procurar una buena calidad de vida hasta el final mediante la utilización de tres herramientas básicas: un adecuado control de síntomas, una correcta información y comunicación con el enfermo y su familia y un buen apoyo psicoemocional. El nacimiento de la Medicina Paliativa, en su concepción actual, es relativamente reciente, por lo que se requiere un cambio de mentalidad de los profesionales sanitarios e implantar un sistema de formación adecuado. Sus recursos son aún insuficientes, no cubriendo a la totalidad de la población subsidiaria. En Medicina Paliativa es frecuente que se plantee la práctica de una sedación profunda y probablemente irreversible como única solución ante un sufrimiento intenso y refractario; esta modalidad de tratamiento en ocasiones anticipa la muerte, eventualidad que puede ser conocida de antemano por el profesional, pero se dice que ésta no es buscada ni deseada, que no es su intención y que sólo es un "efecto secundario"; a esta forma de eutanasia se le denomina comúnmente eutanasia activa indirecta.

Analizadas estas posibilidades, se puede afirmar que las tres primeras modalidades -la muerte en situación de abandono, la muerte con un tratamiento activo hasta el final y la obstinación terapéutica- se consideran inaceptables desde el punto de vista ético por el gran sufrimiento que ocasionan, aunque en los dos últimos supuestos habría que tener en cuenta la opinión y las preferencias del enfermo, verdadero protagonista de la situación, para llegar a esta conclusión.

También se podría asegurar que la atención en el marco de unos cuidados paliativos integrales de calidad es, en principio, si el enfermo tiene la posibilidad de acceder a ellos, la mejor opción cuando está en situación de enfermedad terminal y la muerte es una certeza absoluta.

El dilema ético y la controversia surgen cuando el paciente fallece como consecuencia de una acción u omisión; es decir, cuando en el proceso de la muerte han intervenido una o varias de las siguientes circunstancias: eutanasia pasiva, eutanasia activa indirecta o eutanasia activa directa.

La Legislación de la mayoría de países y la práctica totalidad de los Códigos Deontológicos rechazan, prohiben y penalizan la práctica de la eutanasia activa directa y aceptan, o por 
lo menos justifican o no penalizan, salvo algunas excepciones, la eutanasia pasiva y la activa indirecta. En la situación de enfermedad terminal hay una tendencia a rechazar las acciones cuya intención es producir la muerte, a aceptar las omisiones que la ocasionan y a aceptar también las acciones realizadas con la intención de no ocasionarla, aunque ésta se produzca como consecuencia de su aplicación. El principio ético de autonomía, en determinadas situaciones, cuando supone evitar una acción, adquiere una importancia capital, de modo que se asume que nadie puede obligar a una persona libre, mentalmente competente, psíquicamente sana, sin ninguna clase de coacción y sabiendo perfectamente cuáles son las consecuencias de su elección a recibir un tratamiento que no desea, aunque el resultado de su decisión sea la muerte. Es tan evidente la negativa a justificar la eutanasia activa y a admitir la eutanasia pasiva, que puede darse la siguiente situación paradójica que, si se somete a un proceso de razonamiento, parece totalmente injusta: mientras que cualquier persona, incluso sin encontrarse en situación de enfermedad terminal, que vive o puede seguir viviendo gracias a una técnica o tratamiento, como por ejemplo la amputación de un miembro en caso de una gangrena o la aplicación de hemodiálisis en la insuficiencia renal avanzada, puede decidir morir con sólo rechazarlos y con el amparo de la Ley, esto no puede hacerlo el paciente que no depende de ningún tratamiento o técnica de soporte vital para seguir viviendo, aunque se encuentre incluso en una situación avanzada de enfermedad terminal, con un mayor grado sufrimiento y con una peor calidad de vida. En el primer supuesto, que entraría dentro de la eutanasia pasiva, el principio de autonomía prevalece sobre la obligación ética de mantener la vida, de tal modo que incurriría en un delito quien aplicase estas medidas en contra de la voluntad del enfermo con la intención de salvarle la vida, mientras que en el segundo supuesto, equivalente a una eutanasia activa, los criterios, valores y preferencias del paciente quedan relegados a un segundo plano y el que cometería el delito sería quien los hiciese prevalecer.

A pesar de sus diferencias, se objetiva una característica común en los tres tipos de eutanasia enumerados: la toma de decisiones ha conducido a adoptar una acción u omisión que ha precipitado la muerte del enfermo.

La calificación ética de estas acciones y omisiones dependerá de cada caso en particular y de todo el proceso de razonamiento que ha llevado a adoptar una u otra medida. En la toma de decisiones influyen los valores éticos de la persona responsable de tomar la decisión, sería deseable que también influyesen los del enfermo, y es frecuente que se mezclen una serie de opciones, intenciones y posibles consecuencias, que a veces serán distintas o incluso contradictorias.

La aceptación, con más o menos reservas, de la eutanasia pasiva y de la eutanasia activa indirecta y el rechazo categórico a la eutanasia activa directa se basa en una serie de intuiciones o creencias morales, poco razonadas, compartidas por amplios sectores sociales y convincentes si no se analizan detenidamente. David Hume, hace casi 300 años, afirmaba que las distinciones morales no se derivan de la razón, que la percepción moral de cualquier actuación no siempre tiene que ver con el entendimiento ni con la razón, sino con los gustos o los sentimientos (1).

Ejemplos de estas intuiciones, que no necesariamente resultan éticamente correctas, que son propias de una ética paternalista y sobre las que se basa la legislación de la mayo- ría de los países y los códigos deontológicos para prohibir la eutanasia activa directa, podrían ser los siguientes:

-Equiparar el bien con la vida y el mal con la muerte.

- Provocar la muerte a un enfermo es moralmente menos aceptable que dejarlo morir.

- Dejar que la muerte sobrevenga de manera natural es mejor que anticiparla artificialmente.

- Provocar la muerte de un enfermo de forma intencionada es peor que no evitarla.

Probablemente, en un porcentaje elevado de situaciones estas intuiciones son acertadas y coinciden con una ética que respeta la dignidad de la persona y los derechos humanos fundamentales ya que, por ejemplo, a nadie le debe ser indiferente la injusticia, la provocación anticipada de la muerte y mucho menos el asesinato; además, la eutanasia activa, debido a que supone la pérdida de una vida humana, tampoco debe ser un acto indiferente, debe ser siempre un acontecimiento doloroso y lamentable, incluso para sus más fervientes partidarios. Pero en otras situaciones, quizás las menos, si se analizan detenidamente estas acciones y omisiones, si se toma en consideración las condiciones concretas de cada caso y se reflexiona sobre ellas, enseguida aparecen supuestos en los que, por ejemplo, la eutanasia pasiva puede ser totalmente reprobable y puede enmascarar una discriminación en la utilización de los recursos sanitarios, otros en los que "dejar morir por causas naturales" puede dar lugar a un sufrimiento intenso y cruel, moralmente inaceptable y situaciones en las que se podría considerar correcto provocar o precipitar la muerte por medio de una acción, como ocurre en algunas ocasiones cuando se decide, con el consentimiento del enfermo, aplicar una sedación profunda e irreversible ante un sufrimiento intenso y refractario.

La ética deontológica y la legislación de la mayoría de los países prestan una gran importancia a estas intuiciones morales, a veces poco consistentes y poco razonadas, para catalogar una acción como buena o mala. No se juzga desde una visión ética cada caso en particular y la Justicia tiende a obviar las posibles excepciones por temor a que se puedan cometer abusos. Así, por ejemplo, uno de los pocos argumentos basados en la razón, no en creencias subjetivas o dogmas de fe, que rechazan la despenalización de la eutanasia activa directa es el de la "pendiente resbaladiza", que viene a decir que su legalización o despenalización daría lugar a que también se practique, de forma automática e inevitable, en situaciones que no contempla la ley, como por ejemplo, por motivos sociales, económicos, sin contar con el consentimiento del enfermo, por presión de la familia o incluso del personal sanitario, etc. Es fundamental a la hora de debatir sobre este argumento reconocer que prácticamente todo el mundo asume que el debate sobre la despenalización de la eutanasia se plantea siempre con el requisito asumido de que el paciente mentalmente competente la solicita sin ninguna coacción, estando perfectamente informado de las consecuencias de su decisión y tras agotar todas las medidas paliativas disponibles, apelando a su autonomía y libertad de elección. El argumento de la "pendiente resbaladiza" no indica que la eutanasia activa directa y voluntaria (realizada a petición expresa del paciente) sea moralmente inaceptable, sólo indica que su despenalización abriría una puerta a cometer actos incorrectos. Los tipos de eutanasia activa éticamente inaceptables serían la directa involuntaria (en contra de la voluntad del paciente, lo que equivaldría a un asesinato) y la indirecta no voluntaria (no es 
posible saber si el paciente desea su aplicación o no). Según este argumento, lo que realmente daría consecuencias malas no sería la práctica de la eutanasia activa por si misma, sino legislar a su favor por el riesgo que supone el que se practique en casos no previstos por la ley y poder pasar éstos desapercibidos. Pero prohibir o no despenalizar la eutanasia activa directa no evita el problema porque tanto la eutanasia pasiva como la activa indirecta también pueden practicarse dentro de una mala praxis y ser moralmente inaceptables y también pueden comportar los mismos abusos que se han planteado anteriormente y, sin embargo, dada la intuición moral que acepta que siempre es mejor "dejar morir" que "provocar la muerte", su práctica no se somete al cumplimiento de una serie de requisitos bien detallados ni se penaliza su aplicación.

Existen situaciones oscuras en donde no es posible delimitar claramente la distinción entre acción y omisión, ya que muchas veces la omisión se produce a través de una acción. Por ejemplo, si se interrumpe la nutrición y la hidratación enteral a un paciente diagnosticado de esclerosis múltiple en fase muy avanzada, haciendo caso a sus deseos que formuló a través de unas directrices anticipadas, no cabe ninguna duda de que fallecerá en un periodo corto de tiempo, pero ¿el paciente muere porque debido a sus limitaciones no es capaz de conseguir el alimento o muere porque la acción del médico le causa la muerte?. Parece más razonable la segunda interpretación. En realidad es difícil determinar si el paciente falleció por una acción (eutanasia activa), por una omisión, dejando que la enfermedad siga su curso (eutanasia pasiva) o por una mezcla de ambas. El resultado es el mismo y si se admite que el paciente falleció por una acción, hay que asumir que la eutanasia activa directa puede ser éticamente aceptable, por lo menos en algunas situaciones. Además, se da la circunstancia de que en muchas ocasiones la eutanasia pasiva, el abstenerse de utilizar determinado tratamiento o técnica de soporte vital, no es suficiente para que la muerte se produzca sin sufrimiento, por lo que será necesario aplicar medidas activas que por sí mismas también pueden contribuir a su precipitación; si esto es así, el paciente habrá podido fallecer por una omisión, pero también ha contribuido una acción.

Tampoco resulta sencillo diferenciar entre la forma directa y la forma indirecta de la eutanasia activa porque depende de aspectos totalmente subjetivos como es la intencionalidad del médico. Así, un mismo acto con sus mismas consecuencias puede tener intenciones diametralmente opuestas. Se dice que en la eutanasia activa indirecta la muerte no es intencionada, no es buscada ni deseada, sólo es un efecto secundario. En este tipo de eutanasia, es muy pro- bable que el personal sanitario sepa que causa la muerte del paciente pero no se considera responsable de ella bajo el pretexto de que no era su verdadera intención, argumento poco coherente y falto de consistencia ya que tampoco es su intención evitarla pudiéndolo hacer. Por ejemplo, si se utilizan dosis altas de morfina y midazolam para aliviar un sufrimiento refractario en un paciente con insuficiencia respiratoria severa global y en situación terminal y ocurre un agravamiento previsible de su insuficiencia respiratoria de tal modo que el enfermo entra en coma carbónico, si la intención no es producir la muerte, ¿por qué no se evita retirando estos fármacos y administrando antagonistas opioides como la naloxona y de las benzodiacepinas como el flumazenilo?. Lo razonable y lo correcto en situaciones como ésta, nadie lo debería cuestionar, es permitir la muerte del enfermo, pero hay que admitir que la ocasiona el médico intencionadamente, que tiene la intención de no evitarla, por lo que se puede afirmar que estamos ante una eutanasia activa directa. Es imposible sostener que no se desea la consecuencia de una acción cuando se sabe que tendrá lugar con toda seguridad y no se evita teniendo medios para poderlo hacer.

\section{CONCLUSIONES}

El proceso de la muerte está influenciado por los avances tecnológicos disponibles de modo que, dependiendo del uso que se haga de ellos, ésta podrá acontecer en un momento u otro y de un modo u otro.

La situación ideal sería aquélla que procurase una muerte digna de acuerdo con los deseos del enfermo, libre de sufrimiento y a su tiempo, es decir, sin abreviaciones ni prolongaciones irracionales. La Medicina Paliativa constituye la forma de asistencia más eficaz para conseguir estos objetivos.

En ocasiones, la toma de decisiones lleva a adoptar una serie de medidas, acciones u omisiones, que van a influir en el curso de la enfermedad, precipitando o demorando el momento de la muerte. El personal sanitario, en estos supuestos, debe ser responsable de todos sus actos y, por tanto, de la muerte que provoca o retrasa intencionadamente, pero también de aquélla que no evita habiéndola previsto.

Cuando la muerte es inevitable y existe un sufrimiento intenso y refractario, se debería, al menos, procurar que la persona muera de acuerdo a lo que ella considera que es digno, a sus valores y sus creencias, aceptar su decisión y procurar su cumplimiento. Para que esto sea posible habrá que atreverse a legislar sobre la eutanasia.

\section{Bibliografía}

